\title{
PALABRAS DE CONTENIDO PARANOIDE EN CASTELLANO: FRECUENCIA DE USO, EMOCIONALIDAD Y ESPECIFICIDAD RESPECTO A CONTENIDOS DEPRESIVOS.
}

\author{
MARTA NieTo-Moreno ${ }^{1}$, Gonzalo Hervás ${ }^{2}$ y CARMElo VÁzQuez ${ }^{2}$ \\ ${ }^{\prime}$ Departamento de Psiquiatría, Universidad Autónoma de Madrid \\ ${ }^{2}$ Facultad de Psicología, Universidad Complutense de Madrid
}

\begin{abstract}
Resumen: La investigación en el ámbito de la psicopatología experimental de la paranoia utiliza frecuentemente estímulos verbales. Por esta razón, el disponer de bases de datos validadas de palabras específicamente relacionadas con trastornos paranoides podría constituir una herramienta de gran utilidad para los investigadores. El objetivo de este trabajo fue proporcionar una base de datos en español de palabras relacionadas con la paranoia. El segundo objetivo fue seleccionar palabras específicamente relacionadas con la paranoia y no con la depresión, puesto que palabras de contenido paranoide pueden ser interpretadas de modo depresivo (p.ej., «humillado»). Así, se realizaron dos estudios diferentes. En el estudio 1, el objetivo fue seleccionar una muestra de palabras específicamente relacionadas con contenido paranoide y cuyo contenido depresivo fuera bajo. En el estudio 2, el objetivo fue proporcionar dos índices psicolingüísticos básicos (frecuencia subjetiva de uso y emocionalidad) para la muestra de palabras seleccionadas en el Estudiol. Finalmente, se proporciona un listado de palabras seleccionadas empíricamente siguiendo este procedimiento y se discute la utilidad de disponer de este tipo de estímulos para investigaciones futuras en el ámbito de la psicopatología experimental de trastornos paranoides.
\end{abstract}

Palabras clave: Palabras, estímulos verbales, frecuencia de uso, emocionalidad, paranoia, depresión.

\section{Spanish words with a paranoid content: Frequency of use, emotionality and specificity with respect to depressive contents}

\begin{abstract}
Experimental research on the psychopathology of paranoia frequently uses verbal stimuli. Therefore, the availability of well validated databases of words specifically related to paranoid disorders would be a highly useful tool for researchers. The goal of this study was to provide such database of Spanish paranoia-related words. A second goal was to select words related to paranoia but not to depression as it is possible that words with paranoid content may have a depressive content as well (e.g., 'humiliated'). Thus, we report two different studies. In Study 1, our goal was to select a sample of words specifically related to paranoid contents but not related to depression. In Study 2, our goal was to provide two basic psycholinguistic indexes (subjective frequency of use and emotionality) for the sample of words selected in Study 1. We finally provide the lists of words empirically selected following this procedure and discuss the utility of this type of stimuli for future research on paranoid-related disorders.
\end{abstract}

Keywords: Words, verbal stimuli, frequency of use, emotionality, paranoia, depression.

\section{INTRODUCCIÓN}

La investigación en Psicología clínica experimental emplea con mucha frecuencia tareas que

\footnotetext{
Recibido 19 agosto 2006; aceptado 5 septiembre 2006.

Correspondencia: Carmelo Vázquez, Facultad de Psicología. Universidad Complutense, Campus de Somosaguas, 28223 Madrid. Tfno.:(34) 91-3943131. Fax: (34) 91-3943189. Correo-e: cvazquez@psi.ucm.es.
}

requieren un conocimiento preciso de las características formales de los estímulos empleados. Normalmente estos estímulos suelen ser imágenes o bien estímulos verbales. Desgraciadamen-

Esta investigación fue, en parte, subvencionada por el Ministerio de Ciencia y Tecnología (BSO2003-07516), la Fundación Lafourcade, $\mathrm{y}$ apoyada por una beca FPU del Ministerio de Educación y Cultura (Ref 2001-0786) concedida a Marta Nieto-Moreno. 
te no es frecuente que se controlen con el suficiente rigor las características específicas (p.ej., grado de emocionalidad de las palabras o las imágenes) de las bases estimulares utilizadas.

Por el contrario, en psicología experimental esta preocupación por el control de las variables que pueden tener un efecto espurio en los experimentos, ha recibido una extraordinaria atención por parte de los investigadores (véase la revisión de Proctor y Vu, 1999). Un buen ejemplo de estos esfuerzos es el International Affective Picture System (IAPS) - Lang, Bradley y Cuthbert (1999) - una conocida base de datos de imágenes con datos normativos en diversos parámetros y que, dadas las diferencias culturales que puede haber en los mismos (Sanfeliu y Fernández, 1996), se han hecho estudios específicos normativos con muestras de participantes diferentes países (p.ej., Moltó et al., 1999).

El estudio de las palabras es también un caso ejemplar de los esfuerzos en este sentido. Desde el pionero corpus de Juilland y Chang-Rodríguez (1964) que analizaba la frecuencia de uso de 500.000 palabras, y que es una obra en muchos sentidos obsoleta (Jiménez, Vázquez y Hernangómez, 1998), se han desarrollado posteriormente diversos estudios normativos sobre frecuencia de uso más amplios como el de Alameda y Cuetos (1995) sobre una muestra de 2 millones de palabras. En una reciente revisión efectuada por Pérez, Campoy y Navalón (2001), se han contabilizado y analizado las características de 74 bases de datos diferentes, realizadas por investigadores de diversos países hispanohablantes, con normas para diversos materiales (básicamente palabras en castellano, aunque también incluye algunos estudios realizados con dibujos). Quizás el trabajo más ambicioso en esta línea es la base denominada LEXESP (Sebastián, Martí, Carreiras, y Cuetos, 2000) que incluye índices objetivos (frecuencia de uso, número de sílabas y una serie de índices morfosintácticos como forma, lema, máscara y categoría) y subjetivos (familiaridad, concreción e imaginabilidad) de un corpus de 5.020.930 palabras extraídas de textos escritos. Otros trabajos proporcionan datos sobre asociaciones entre palabras (p.ej., Alonso y Díez, 2000; Callejas, Correa, Lupiáñez y Tudela, 2003), o incluso características de determinadas submuestras de bases léxicas como el LEXESP (p.ej., palabras de 4 letras: Alameda y Cuetos, 2000; valor afectivo de una serie de 478 palabras: Redondo, Fraga, Comesaña y Perea, 2005) o incluso submuestras extraídas del Diccionario de la Real Academia (p.ej., características como frecuencia y longitud de palabras entre 3 y 16 letras: Pérez, Alameda y Cuetos, 2003).

Aunque en muchos estudios experimentales con estímulos verbales o de imágenes se efectúan pequeños estudios piloto previos para controlar algunas de estas variables indicadas (véase por ejemplo Calvo y Castillo, 2005), el hecho de contar con bases disponibles previas puede facilitar de modo sustancial la tarea de los investigadores $y$ tener marcos de referencia comunes que permitan una mayor generalización de los resultados.

¿Cuál es la utilidad potencial de disponer de estímulos normativizados en psicología clínica? La investigación experimental sobre procesos cognitivos en Psicopatología ha tenido un crecimiento espectacular en los últimos años en áreas como la depresión y la ansiedad (Mathews y MacLeod, 2005) pero también en temas como los trastornos alimentarios (Cooper, 2005), o incluso en el terreno de las psicosis (Bentall, 2003). En muchos de estos casos, la investigación se ha valido de tareas que requieren el uso de estímulos verbales (tareas de escucha dicótica, de decisión léxica, de Stroop emocional, de memoria implícita, de anticipación semántica, de codificación autorreferente, dot probe, etc.). Sin embargo, el empleo de material verbal supone una serie de notables dificultades experimentales (ver Jiménez et al., 1998). Por ejemplo, si se utilizan palabras, éstas difieren entre sí no sólo en su significado objetivo (denotación), su longitud, o su número de fonemas, sino en muchos otros parámetros de potencial importancia: significado subjetivo (connotación), frecuencia objetiva y/o subjetiva de uso, facilidad de comprensión, emocionalidad, importancia personal, autodescriptividad, imaginabilidad, etc. Esto hace que el uso de palabras en la investigación sea un asunto realmente delicado y de necesario aunque difícil control (Sanz, 1995).

La disponibilidad de nuevas bases de palabras normativizadas en español como, por ejemplo, la citada LEXESP, puede hacer cada vez más sencillo el empleo de estímulos verbales con un correcto control de ciertos parámetros. Pero aún así, los investigadores de temas psicopatológicos 
Tabla 1. Estudios normativos sobre palabras en español relacionadas con problemas psicológicos

\begin{tabular}{|c|c|c|c|c|c|}
\hline Autor & Tema & Muestras & Corpus & Categorías & Resultados \\
\hline $\operatorname{Sanz}(1995)^{\wedge}$ & $\begin{array}{l}\text { Ansiedad } \\
\text { social } \\
\text { y depresión }\end{array}$ & $\begin{array}{l}99 \text { estudiantes de } \\
\text { Psicología ( } 84 \% \\
\text { mujeres). Edad } \\
\text { media: } 21.3 \text { años }\end{array}$ & $\begin{array}{l}96 \text { adjetivos positivos y } \\
\text { negativos seleccionados a } \\
\text { partir de } 132 \text { extraidos de tests } \\
\text { e inventarios de personalidad } \\
\text { general: } \\
\text { - Negativos (ansiedad): } n=12 \\
\text { - Negativos (depresión): } n=12 \\
\text { - Positivos (ansiedad): } n=12 \\
\text { - Positivos (depresión): } n=12 \\
\text { - Neutrales positivos: } n=36 \\
\text { - Neutrales negativos: }=12\end{array}$ & $\begin{array}{l}\text { Evaluación de los participantes } \\
\text { en escalas Likert (0-10): } \\
\text { Descriptividad de depresión. } \\
\text { Descriptividad de ansiedad. } \\
\text { Emocionalidad } \\
\text { Recuencia de uso } \\
\text { Frecuencia subjetiva } \\
\text { Imaginabilidad } \\
\text { Autodescriptividad } \\
\text { Importancia personal }\end{array}$ & $\begin{array}{l}\text { Datos normativos sólo por } \\
\text { grupos de palabras } \\
\text { pertenecientes a cada } \\
\text { categoria. } \\
\text { Datos sobre adjetivos } \\
\text { empiricamente relacionados } \\
\text { (correlaciones parciales) con } \\
\text { Ansiedad social, Depresión, o } \\
\text { ambos trastornos }\end{array}$ \\
\hline $\begin{array}{l}\text { Ruipérez y } \\
\text { Belloch (1997) }\end{array}$ & $\begin{array}{l}\text { Ansiedad y } \\
\text { depresión }\end{array}$ & $\begin{array}{l}10 \text { psicólogos y } \\
\text { psiquiatras que } \\
\text { evalúan las } \\
\text { palabras como } \\
\text { expertos }\end{array}$ & $\begin{array}{l}75 \text { adjetivos seleccionados a } \\
\text { partir de una lista de } 200 \\
\text { palabras utilizadas en } \\
\text { investigaciones publicadas: } \\
\text { - Negativos (ansiedad y } \\
\text { depresión) ( } \mathrm{n}=25) \\
\text { - Positivos }(\mathrm{n}=25) \\
\text { - Neutros ( } \mathrm{n}=25)\end{array}$ & $\begin{array}{l}\text { Evaluación de los participantes } \\
\text { en escalas Likert ( } 0 \text {-10): } \\
\text { Capacidad de la palabra para } \\
\text { «describir características } \\
\text { emocionales») relacionadas con } \\
\text { rasgos depresivos, positivos, y } \\
\text { neutros. }\end{array}$ & $\begin{array}{l}\text { Sin datos normativos. Se } \\
\text { publica la lista de adjetivos que } \\
\text { obtuvieron una puntuación } \geq 6 \\
\text { en una categoria } y \leq 3 \text { en las } \\
\text { otras dos. }\end{array}$ \\
\hline $\begin{array}{l}\text { Blanch y Baños } \\
\text { (1996) }\end{array}$ & $\begin{array}{l}\text { Depresión y } \\
\text { ansiedad }\end{array}$ & $\begin{array}{l}212 \text { adultos ( } 75 \% \\
\text { mujeres). Edad } \\
\text { media: } 34.4 \text { años } \\
\text { (rango: } 17-62 \text { ) }\end{array}$ & $\begin{array}{l}125 \text { sustantivos y adjetivos } \\
\text { relacionados con ansiedad y } \\
\text { depresión. Extraidos de } \\
\text { cuestionarios y tests. }\end{array}$ & $\begin{array}{l}\text { Evaluación de los participantes } \\
\text { en escalas Likert (0-10): } \\
\text { 1) Frecuencia de uso subjetiva } \\
\text { 2) Comprensión } \\
\text { 3) Imaginabilidad } \\
\text { 4) Descriptividad de ansiedad } \\
\text { 5) Descriptividad de depresión }\end{array}$ & $\begin{array}{l}\text { Datos para cada palabra por } \\
\text { sexo, grupos de edad y nivel } \\
\text { de estudjos }\end{array}$ \\
\hline $\begin{array}{l}\text { Pons y Perpiñáa } \\
\text { (1996) }\end{array}$ & $\begin{array}{l}\text { Trastomos } \\
\text { alimentarios }\end{array}$ & $\begin{array}{l}100 \text { jóvenes sin } \\
\text { trastomos } \\
\text { alimentarios ( } 90 \% \\
\text { mujeres). Edad } \\
\text { media: } 18.9 \text { años } \\
\text { (rango: } 14-24 \text { ) }\end{array}$ & $\begin{array}{l}\text { Palabras relacionadas con: } \\
\text { Comida }(n=56) \\
\text { Cuerpo }(n=53) \\
\text { Artículos del hogar }(n=56) \\
\text { Plantas }(n=50)\end{array}$ & $\begin{array}{l}\text { Evaluación de los participantes } \\
\text { en escalas Likert (0-10): } \\
\text { 1) Pertenencia a la categoria } \\
\text { 2) Frecuencia de uso } \\
\text { 3) Familiaridad }\end{array}$ & $\begin{array}{l}\text { Datos normativos para cada } \\
\text { palabra }\end{array}$ \\
\hline $\begin{array}{l}\text { Jiménez, } \\
\text { Vázquez y } \\
\text { Hernangómez } \\
\text { (1998) }\end{array}$ & $\begin{array}{l}\text { Depresión } \\
\text { (pura) (no } \\
\text { ansiosa)) }\end{array}$ & $\begin{array}{l}172 \text { estudiantes de } \\
\text { Psicologia ( } 84 \% \\
\text { mujeres). Edad } \\
\text { media: } 21.5 \text { años } \\
\text { (rango: } 19-47 \text { ) }\end{array}$ & $\begin{array}{l}\text { Adjetivos relacionados con } \\
\text { sintomas DSM-IV de la } \\
\text { depresión (y sinónimos y } \\
\text { antónimos): } \\
\text { - } 98 \text { negativos } \\
\text { - } 60 \text { positivos } \\
\text { Adjetivos neutros }(n=92)\end{array}$ & $\begin{array}{l}\text { Evaluación de los participantes } \\
\text { en escalas Likert: } \\
\text { 1) Autorreferencia } \\
\text { 2) Emocional idad (positiva o } \\
\text { negativa) } \\
\text { 3) Frecuencia de uso }\end{array}$ & $\begin{array}{l}\text { Datos normativos para cada } \\
\text { palabra en emocionalidad y } \\
\text { frecuencia. } \\
\text { Se distingue entre adjetivos } \\
\text { descriptivos de depresión pura } \\
\text { y depresión-ansiedad (estado } \\
\text { mixto) }\end{array}$ \\
\hline $\begin{array}{l}\text { Castañeiras y } \\
\text { Belloch }(2000)\end{array}$ & Hipocondria & $\begin{array}{l}\text { 186 estudiantes de } \\
\text { Psicología ( } 83 \% \\
\text { mujeres). Edad } \\
\text { media: } 22.6 \text { años } \\
\text { (rango: } 20-47 \text { ) }\end{array}$ & $\begin{array}{l}77 \text { palabras relacionadas con } \\
\text { temas de enfermedad } \\
\text { (sensaciones fisicas } \\
\text { o sintomas corporales) }\end{array}$ & $\begin{array}{l}\text { Evaluación de los participantes } \\
\text { en escalas Likert: } \\
\text { 1) Frecuencia de uso } \\
\text { 2) Emocionalidad } \\
\text { 3) Imaginabilidad } \\
\text { 4) Descriptividad de } \\
\text { enfermedad }\end{array}$ & $\begin{array}{l}\text { Datos normativos para cada } \\
\text { palabra. }\end{array}$ \\
\hline
\end{tabular}

${ }^{\text {a }}$ En el estudio se emplean varias muestras sucesivas con diferentes tamaños muestrales. 
específicos pueden tener problemas para hallar suficientes palabras de un dominio léxico específico (i.e. trastornos de alimentación). Esta necesidad ha promovido una serie de investigaciones en las que se proporcionan datos normativos de palabras relacionadas en castellano con algunos trastornos, lo que sin duda supone ya un notable avance en este sentido. En la Tabla 1 ofrecemos un resumen de las características disponibles de los estudios que conocemos disponibles sobre dichas bases de datos hasta la actualidad.

Como puede verse en la citada tabla, estos estudios tienen una metodología variada y reflejan, de hecho, la ausencia de unanimidad sobre el mejor procedimiento de selección de palabras para su uso en la investigación (véase una revisión en Sanz, 1995). A veces la selección de estimulos experimentales se basa en el criterio personal de los investigadores (p.ej., Foa, McNally y Murdock, 1989) o en estímulos seleccionados por una serie de expertos (p.ej., Blanch y Baños, 1997). En otros estudios más controlados los investigadores suelen construir las bases de datos de un modo empírico (Jiménez et al., 1998) que, a veces, consiste en recurrir a bases de datos normativizadas o bien en efectuar algún estudio piloto previo a pequeña escala con las palabras seleccionadas (p.ej., Calvo y Castillo 2005). Tampoco existe acuerdo sobre la selección final de palabras. Dada la variabilidad en la frecuencia de uso, connotación, emocionalidad, etc. de las palabras, algunos autores han propuesto que las técnicas idiográficas serían preferibles (McNally, Amir, Louro, Lukach, Rieman, y Calamari, 1994) de modo que las palabras a utilizar en cada estudio serían seleccionadas para cada uno de los participantes a partir de una base de datos inicial. No obstante este método idiográfico también plantea problemas. Además de que cada sujeto estaría expuesto a estímulos físicamente distintos, no siempre es fácil disponer de un conjunto inicial de palabras suficientemente grande, especialmente en investigaciones clínicas, como para que cada sujeto esté expuesto, finalmente, a un número suficiente de estímulos experimentales. Asimismo, con esta estrategia particularizada para cada individuo pueden acentuarse indeseables efectos de priming pues los estímulos que serán utilizados en la tarea experimental han de ser administrados previamente al participante para efectuar su selección. Una dificultad menor, pero también a tener en cuenta, es que este procedimiento individualizado alarga más el tiempo de cada experimento. No obstante, si se pudiera disponer de un banco de datos muy amplio de palabras, los problemas de esta estrategia quedarían algo aminorados.

La investigación psicopatológica ha estado guiada por la idea de que los trastornos mentales (básicamente depresión y ansiedad) difieren entre sí más por los contenidos de lo procesado (p.ej., sesgos de atención o de memoria hacia estímulos relacionados con temas de pérdida en el caso de la depresión y hacia estímulos relacionados con temas de amenaza en el caso de la ansiedad) que por los propios procesos implicados. Aunque esta hipótesis de la especificidad del contenido (Beck, Brown, Steer, Eidelson y Riskind, 1987) ha recibido críticas en estos últimos años (p.ej., Beck y Perkins, 2001; Vázquez y Cameron, 1997), abrió la necesidad, aún hoy relevante, de intentar emplear estímulos lo más específicamente relacionados con un trastorno determinado. Así, por ejemplo, la investigación con procesos mnésicos en la depresión puso muy pronto de manifiesto que el mejor modo de activar esquemas depresivos era emplear palabras con un contenido específico relevante a la depresión y no cualquier tipo de estímulo de contenido negativo general (Greenberg, Vázquez, y Alloy, 1988). Aunque esto parezca bastante obvio, ha sido un aspecto sorprendentemente descuidado en muchos estudios sobre cognición y psicopatología (Sanz, 1995).

En este mismo marco de la psicopatología y psicología clínica experimental, el estudio del delirio ha recibido una especial atención en estos últimos años. Los modelos cognitivos actuales de los delirios, especialmente de los delirios paranoides, asumen que las ideas delirantes son semejantes a las creencias «normales» en cuanto a los procesos cognitivos y emocionales implicados en su formación y mantenimiento (Bentall, 1990; Diez-Alegría, Nieto y Vázquez, 2001; Diez-Alegría y Vázquez, en prensa). Numerosos trabajos han aplicado los modelos de procesamiento de información en su estudio, empleando las mismas tareas y tipo de material experimental que las utilizadas en los estudios de procesos cognitivos implicados en otros trastornos como la depresión o la ansiedad (p.ej., tarea Stroop emo- 
cional, tareas de escucha dicótica, tareas de recuerdo incidental, pruebas de razonamiento causal, etc.; ver revisión en Bentall, 2003). La utilización de material experimental específico y relevante para individuos con ideación delirante paranoide (i.e. contenido de amenaza interpersonal hacia el self), en su mayoría estímulos verbales, ha permitido identificar sesgos perceptivos e inferenciales en el procesamiento de información de contenido amenazante (p.ej., Bentall, 2003; Blackwood, Howard, Bentall y Murray, 2001).

En nuestro país se han realizado investigaciones empleando estímulos verbales en castellano usando tareas experimentales con pacientes psicóticos, como los estudios de Ruiz-Vargas y Ochoa (1987) en el ámbito de la memoria verbal, o de Vázquez et al. (1990) con una tarea de escucha dicótica. Hasta donde sabemos no se ha efectuado ningún estudio para validar específicamente palabras relacionadas con las psicosis y, más en concreto, con delirios de tipo persecutorio. Aunque la mera traducción al castellano de material verbal de contenido amenazante empleado en otros estudios sería una estrategia parcialmente válida, un análisis más exhaustivo de este tipo de trabajos nos permite detectar un alto grado de solapamiento de los estímulos verbales en relación a diferentes trastornos. Esto puede dificultar el estudio de procesos cognitivos implícitos específicos a un trastorno en particular que sólo se activarían ante material emocionalmente relevante. Por ejemplo, algunas de las palabras empleadas en estudios previos como estímulos experimentales relacionados con el constructo paranoidismo o de delirio de tipo persecutorio (Kinderman, Prince, Waller y Peters, 2003) también estaban seleccionadas en el estudio de Jiménez et al. (1998) como adjetivos de contenido autorreferente depresivo (en concreto, las palabras aislado, decaído, estúpido, fracasado, impotente, inútil, solitario y solo).

En definitiva, nos pareció necesario disponer de estímulos experimentales específicos y válidos para el estudio experimental de procesos cognitivos implicados en la ideación de contenido persecutorio. El objetivo final del trabajo fue elaborar una lista empíricamente seleccionada de palabras relacionados con ideación paranoide o con ideación de amenaza interpersonal que proporcione además información sobre su frecuen- cia de uso en castellano y su grado de emocionalidad para su posterior utilización como estímulos experimentales en estudios en los que sea necesario utilizar palabras con ese tipo de contenido. En concreto, con nuestro trabajo hemos pretendido cubrir varios fines: 1) Elaborar una lista suficientemente rigurosa y válida de palabras que puedan ser utilizadas como estímulos experimentales en estudios de los procesos cognitivos relacionados con ideación de contenido persecutorio. 2) Determinar la especificidad de contenido amenazante o persecutorio de los estímulos experimentales para, finalmente, disponer de estímulos experimentales de contenido persecutorio «puro» con respecto a estímulos de contenido depresivo (para una revisión de la hipótesis de que paranoia y depresión son dos condiciones psicopatológicamente semejantes, véase Bentall, 2003). Para la consecución de los objetivos propuestos se realizaron dos estudios diferentes cuya metodología y resultados respectivos se describen en las siguientes secciones.

\section{ESTUDIO 1}

Los objetivos de este primer estudio fueron: 1) estudiar en qué medida las palabras presentados en un listado creado ad hoc - Cuestionario de Palabras de Amenaza Interpersonal (Nieto, Hervás y Vázquez, 2006 - ver sección Instrumentos) - hacía referencia al constructo amenaza interpersonal; 2) determinar el grado en que dichas palabras conllevaban contenido amenazante y depresivo, con el fin de permitir una selección precisa de palabras amenazantes "puras» (i.e. con bajo grado de contenido depresivo) y palabras depresivas «puras» (i.e. con bajo grado de contenido amenazante).

\section{Participantes}

Participaron en este primer estudio 90 estudiantes de Psicología (20 hombres y 70 mujeres) cuya edad media fue 22,13 años $(D T=1,36)$. Los participantes se ofrecieron voluntariamente dentro de una clase de la Facultad de Psicología. 


\section{Instrumentos}

Se aplicó el Cuestionario de Palabras de Amenaza Interpersonal (Nieto, Hervás y Vázquez, 2006). El listado estuvo compuesto por 139 palabras ( 79 de contenido amenazante y 60 de contenido depresivo). Las palabras de contenido depresivo fueron extraídas del estudio de validación previo de Jiménez et al. (1998). El objetivo de su inclusión fue determinar en qué medida cada uno de ellos pudiera tener un contenido amenazante, algo esperable si tenemos en cuenta las teorías que proponen que los pacientes con delirios persecutorios tienen esquemas depresivos latentes (Bentall, 2003). Para seleccionar las palabras de contenido amenazante se elaboró una lista de 140 palabras que, desde un punto de vista amplio, estaban relacionadas con el constructo de amenaza interpersonal. Las fuentes de procedencia de las palabras fueron varias: 1) El conjunto de palabras empleadas en estudios previos sobre ideación paranoide (Kinderman et al., 2003); 2) palabras relacionadas con la amenaza social usadas por Quero, Baños y Botella (2001); 3) diccionario de sinónimos; 4) palabras amenazantes relacionadas con el trastorno de pánico (en el que claramente existen componentes amenazantes) usadas por Quero y cols. (2001).

De estas 140 palabras iniciales de amenaza los investigadores seleccionaron sólo aquéllas que hacian referencia a contenidos interpersonales (79 palabras) y aunque se seleccionaron predominantemente adjetivos, pues tienen un mayor potencial para activar connotaciones y elementos valorativos, se incluyeron también sustantivos y verbos procedentes de trabajos experimentales previos sobre paranoidismo (Kinderman et al., 2003).

\section{Procedimiento}

Las 139 palabras seleccionadas fueron presentadas, ordenadas alfabéticamente, en el Cuestionario de Palabras de Amenaza Interpersonal. Se pidió a los participantes que evaluasen en qué medida el contenido semántico de cada una de las palabras tenía un significado «amenazante» (de tipo paranoide), empleando para ello una escala tipo Likert de 0 (= Nada amenazante) a 10 (=
Totalmente amenazante). Las instrucciones textualmente indicaban lo siguiente: «Estamos realizando un estudio sobre tipos de palabras que en nuestro idioma pueden tener un significado amenazante. Por significado amenazante entendemos cualquier alusión que el contenido semántico de la palabra haga al perjuicio / amenaza o que atente contra la autoestima y la integridad fisica, moral e interpersonal de un individuo. A continuación presentamos una lista de palabras para evaluar dicho contenido en cada una».

Una vez completado dicho cuestionario, se presentó a los participantes otra lista de palabras idéntica en la que valoraron en qué grado la característica a la que hacía referencia cada una de las palabras tenía un contenido depresivo. Se realizaron dos versiones paralelas que diferían en el género de las palabras con el objetivo de que los hombres y mujeres participantes pudieran hacer valoraciones concordantes con su propio género.

\section{Resultados}

Se efectuó un análisis descriptivo de los datos, obteniendo el grado medio de contenido amenazante y depresivo para cada palabra. El resultado de este análisis se muestra en el Anexo I. Para la consecución del segundo objetivo en este primer estudio, esto es, elaborar una lista con las palabras de mayor grado de especificidad en cuanto a contenido paranoide por una parte y de contenido depresivo por otro lado, el procedimiento seguido fue el siguiente: en primer lugar, se seleccionaron las 70 palabras con mayor grado medio de amenaza y las 70 palabras con mayor grado medio de contenido depresivo. Posteriormente, y para cada palabra, se calculó la diferencia entre las puntuaciones medias en grado de amenaza y de depresión. De esta forma, obtuvimos un índice de especificidad en cuanto al contenido amenazante vs. depresivo. La selección final de palabras «amenazantes» y «depresivas» fue realizada atendiendo ambos criterios, es decir, aquéllas palabras con mayores puntuaciones en amenaza y depresión y que, además, su diferencia de medias en ambos constructos fuera superior a 2 puntos. Estas palabras fueron (ordenadas por grado de especificidad) las que figuran en la Tabla 2. 


\section{ESTUDIO 2}

Los objetivos de este estudio fueron determinar la emocionalidad y frecuencia subjetiva de uso de las palabras del Cuestionario de Palabras de Amenaza Interpersonal.

\section{Participantes}

El Cuestionario de Palabras de Amenaza Interpersonal fue completado por 106 estudiantes de psicología ( 18 hombres y 88 mujeres; edad media $=20,98 ; D T=1,5)$. Los participantes se ofrecieron voluntariamente dentro de una clase de la Facultad de Psicología.

\section{Procedimiento}

El protocolo de evaluación fue administrado de forma colectiva y anónima. Las instrucciones proporcionadas a los participantes en este segundo estudio fueron las siguientes: "Valora en qué grado la característica a la que hace referencia este adjetivo es para ti negativa en una escala de 0 a 10 , siendo $0=$ Nada negativa y $10=$ Totalmente negativa. A continuación, valora con qué frecuencia utilizas este adjetivo en tu vida en una escala de 0 a 10 , siendo $0=$ Nunca y $10=$ Constantemente». La duración total de su aplicación fue aproximadamente 30 minutos.

Tabla 2. Listado de palabras seleccionadas con mayor grado de especificidad en cuanto a contenido amenazante y contenido depresivo.

\begin{tabular}{|c|c|c|c|}
\hline $\begin{array}{l}\text { Contenido } \\
\text { Amenazante }\end{array}$ & $\begin{array}{c}\text { Indice de } \\
\text { especificidad }\end{array}$ & $\begin{array}{l}\text { Contenido } \\
\text { Depresivo }\end{array}$ & $\begin{array}{c}\text { Indice de } \\
\text { especificidad }\end{array}$ \\
\hline Asesinar & 5,38 & Deprimido/a & $-4,98$ \\
\hline Matar & 5,34 & Decaido/a & $-4,88$ \\
\hline Asesinado/a & 5,28 & Desganado/a & $-4,64$ \\
\hline Atracado/a & 4,43 & Apenado/a & $-4,51$ \\
\hline Espiado/a & 4,22 & Apesadumbrado/a & $-4,51$ \\
\hline Atacado/a & 4,10 & Apagado/a & $-4,29$ \\
\hline Acosado/a & 3,87 & Alicaído/a & $-4,18$ \\
\hline Sangre & 3,85 & Aislado/a & $-3,9$ \\
\hline Secuestrado/a & 3,83 & Desilusionado/a & $-3,89$ \\
\hline Amenazado/a & 3,72 & Desmotivado/a & $-3,84$ \\
\hline Vigilado/a & 3,55 & Triste & $-3,82$ \\
\hline Malo/a & 3,39 & Desesperanzado/a & $-3,8$ \\
\hline Estafado/a & 3,35 & Abatido/a & $-3,8$ \\
\hline Perseguido/a & 3,35 & Desconsolado/a & $-3,76$ \\
\hline Chantajeado/a & 3,09 & Pesimista & $-3,5$ \\
\hline Capturado/a & 3,08 & Desolado/a & $-3,15$ \\
\hline Acusado/a & 2,86 & Solitario/a & $-3,11$ \\
\hline Condenado/a & 2,85 & Inapetente & $-3,01$ \\
\hline Controlado/a & 2,62 & Solo/a & $-2,98$ \\
\hline Manipulado/a & 2,41 & Vacío/a & $-2,77$ \\
\hline Atemorizado/a & 2,40 & Derrotado/a & $-2,74$ \\
\hline Asediado/a & 2,35 & Desgraciado/a & $-2,68$ \\
\hline Boicoteado/a & 2,26 & Desalentado/a & $-2,61$ \\
\hline Apresado/a & 2,23 & Derrumbado/a & $-2,54$ \\
\hline Castigado/a & 2,07 & Infeliz & $-2,51$ \\
\hline \multirow[t]{5}{*}{ Calumniado/a } & 2,00 & Desamparado/a & $-2,47$ \\
\hline & & Aburrido/a & $-2,42$ \\
\hline & & Inactivo/a & $-2,31$ \\
\hline & & Abandonado/a & $-2,26$ \\
\hline & & Hundido/a & $-2,07$ \\
\hline
\end{tabular}




\section{Resultados}

El análisis descriptivo de los datos en las variables Emocionalidad y Frecuencia subjetiva de uso para cada una de las palabras del cuestionario se muestra en el Anexo II.

\section{DISCUSIÓN}

Estos dos estudios tenían como objetivo general estudiar las propiedades de dos listas de palabras -depresivas y amenazantes (de tipo persecutorio) - para posibilitar su uso en investigación de forma controlada. En primer lugar, y dado que determinadas palabras podían tener cierto solapamiento de contenido en cuanto a su alusión a lo amenazante y a lo depresivo, se diseñó un estudio inicial para poder localizar las palabras con mayor especificidad de contenido, es decir, aquellas palabras de alto contenido depresivo y bajo amenazante por una parte, y las de alto contenido amenazante y bajo depresivo, por otra.

Este primer estudio, arrojó algunos datos interesantes; por ejemplo, palabras como «humillado» o «fracasado» pueden hacer referencia tanto a contenidos de amenaza como a contenidos de carácter más depresivo. Otras palabras como "condenado", extraída del listado de palabras depresivas «puras» previamente validado en nuestro país por Jiménez et al. (1998) emerge como uno de los estímulos experimentales con mayor contenido amenazante. En esta línea, la especificidad de estímulos amenazantes empleados en estudios realizados en otros países (i.e. Kinderman et al., 2003) resulta dudosa a la luz de nuestros resultados; valgan como ejemplo las palabras «lisiado» cuya puntuación media fue similar tanto para el constructo amenaza como para el depresión, o «abandonado", cuya valoración fue superior en el contenido depresivo. En consecuencia, conviene alertar a los investigadores respecto a la especificidad del material experimental empleado en trabajos cuyo fin es la activación de contenidos implícitos y sesgos en el procesamiento de la información ligados a trastornos específicos mediante material experi- mental como el aquí citado. Por otro lado, el solapamiento de contenidos depresivos y amenazantes (de tipo interpersonal, persecutorio o paranoide), no es sorprendente. A pesar de las notables y aparentes diferencias clínicas entre la depresión y la ideación paranoide clínica, es posible que ambas compartan constructos implícitos y procesos que en determinadas ocasiones son difíciles de delimitar (Bentall, 2003), algo extensible también a los procesos comunes implicados entre, por ejemplo, delirios paranoides y ansiedad (Freeman, Garety, Kuipers, Fowler y Bebbington, 2002). Es posible, además, que la connotación subjetiva de palabras como «humillado» (por continuar con el ejemplo anterior) esté mediada por procesos ligados, por ejemplo, al razonamiento inferencial, que podrían diferenciar el carácter puramente depresivo o persecutorio de algunos de los estímulos presentados (en el caso de «humillado», un locus atribucional interno vs. un locus atribucional externo en el que estarían implicadas otras personas, podría determinar un contenido u otro). En cualquier caso, éstas son hipótesis planteadas que requieren estudios futuros para su constatación.

Los datos presentados en el segundo estudio permiten construir listados de palabras de forma que se puedan controlar la frecuencia subjetiva de uso así como su emocionalidad, quizá las dos propiedades más usualmente controladas en la investigación. La combinación de los datos ofrecidos por ambos estudios permite por ejemplo construir listados de parejas de palabras (depresivas-amenazantes) comparables en número de letras, y con similar emocionalidad y frecuencia de uso. Asimismo, es posible combinar los resultados de estos estudios con otros previos y hacer listados de pares de palabras incluyendo palabras con otro tipo de contenido (neutras, positivas, de carácter ansioso, etc).

No obstante, hay que resaltar la necesidad de estudios futuros para determinar la especificidad del listado de palabras presentado en este trabajo respecto a contenidos ligados a la ansiedad (por citar un ejemplo) así como para analizar otras variables relevantes para la investigación como lo es la imaginabilidad. Otro aspecto a tener en cuenta del presente trabajo es la población utilizada, universitaria, por lo que 
habría que tener cautelas a la hora de generalizar el uso de los listados que aquí se presentan en otro tipo de muestras. Además, y puesto que su participación fue voluntaria, no se controló el potencial sesgo de selección de los participantes, siendo posible que aquellos individuos con mayor ideación paranoide (subclínica) o con rasgos paranoides de la personalidad rechazaran participar en el estudio, pudiendo estar infravaloradas las puntuaciones obtenidas en cuanto al grado de emocionalidad y contenido de amenaza de las palabras. En todo caso, el objetivo del presente trabajo se centraba en delimitar el campo semántico de determinadas palabras en el lenguaje natural. Finalmente, cabe resaltar que en este trabajo la mayor parte de los participantes fueron mujeres. Aunque los estudios sobre razonamiento paranoide y procesamiento de la información no parecen indicar que existan diferencias en función del género de los participantes, estudios futuros habrán de tener en cuenta la potencial influencia de esta variable en los estudios experimental.

A modo de conclusión, pensamos que trabajos como el aquí presentado, permiten el empleo de material experimental riguroso y específico para estudios en los que se analizan procesos implicados en el mantenimiento y formación de ideación delirante (subclínica o clínica), y que finalmente permiten el estudio de síntomas «olvidados» durante mucho tiempo por la psicología clínica experimental, y que en los últimos años han propiciado el desarrollo de modelos psicopatológicos explicativos (p.ej., Bentall, Corcoran, Howard, et al., 2001; Freeman et al., 2002) que han hecho factible el desarrollo de estrategias de intervención (p.ej. Perona, Cuevas-Yust, Vallina y Lemos, 2003; Nieto y Sotoca, 2005) en el campo de la Psicología clínica.

\section{REFERENCIAS}

Alameda, J.R. y Cuetos, F. (1995). Diccionario de frecuencias de las unidades lingüísticas del castellano. Oviedo: Servicio de Publicaciones de la Universidad de Oviedo.

Alameda, J.R. y Cuetos (2000). Incidencia de la vecindad ortográfica en el reconocimiento de palabras. Revista de Psicologia General y Aplicada, 53, 85-107.
Alonso, M.A. y Díez, E. (2000). Efectos del olvido dirigido en el método de las palabras: Una comparación entre pruebas directas e indirectas de memoria. Psicológica, 21, 1-22.

Beck, A.T., Brown, G., Steer, R.A., Eidelson, J.I. y Riskind, J.H. (1987). Differentiating anxiety and depression: A test of the cognitive content-specificity hypothesis. Journal of Abnormal Psychology, 96, 179-183.

Beck, R. y Perkins, T. S. (2001). Cognitive content-specificity for anxiety and depression: A meta-analysis. Cognitive Therapy and Research, 25, 651-663.

Bentall, R.P. (1990). The syndromes and symptoms of psychosis: Or why you can't play twenty questions with the concept of schizophrenia and hope to win. En R. Bentall (Ed), Reconstructing Schizophrenia. London: Routledge.

Bentall, R.P. (2003). Madness explained: Psychosis and human nature. London: Allen Lane.

Bentall, R.P., Corcoran, R., Howard, R., Blackwood, N. y Kinderman, P. (2001). Persecutory delusions: A review and theoretical integration. Clinical Psychology Review, 21, 1143-1192.

Blackwood, N., Howard, R., Bentall, R. y Murray, R. (2001). Cognitive neuropsychiatric models of persecutory delusions. American Journal of Psychiatry, 158 , 527-539.

Blanch, M. T. y Baños, R. (1996). Estimulos verbales y trastornos emocionales: un estudio sobre palabras con contenido emocional. Revista de Psicopatología y Psicología Clínica, 1, 137-157.

Callejas, A., Correa, A., Lupiáñez, J., y Tudela, P. (2003). Normas asociativas intracategoriales para 612 palabras de seis categorías semánticas en español. Psicológica, 24, 185-214.

Calvo, M.G. y Castillo, M.D. (2005). Processing of Threat-related Information Outside the Focus of Visual Attention. Spanish Journal of Psychology. 8, 3-11.

Castañeiras, C., y Belloch, A. (2000). Estímulos verbales asociados a hipocondría. Revista de Psicopatologia y Psicología Clínica, 5, 103-113.

Cooper, M. (2005). A developmental vulnerability-stress model of eating disorders: A cognitive approach. In B.L. Hankin y J.R.Z. Abela (Eds.). Development of psychopathology: A vulnerability-stress perspective (pp. 328-354). New York: Sage.

Díez-Alegría, C., Nieto, M. y Vázquez, C. (2001). Estudio del delirio desde una perspectiva cognitiva: Una revisión de modelos teóricos. Psicopatología Clínica Legal y Forense, 1, 51-65

Díez-Alegria, C., y Vázquez, C (2006, en prensa). Teorias cognitivas del delirio. Apuntes de Psicologia.

Foa, E. B., McNally, R. J., y Murdock, T. B. (1989). Anxious mood and memory. Behaviour Research and Therapy, 27, 141-147. 
Freeman, D., Garety, P., Kuipers, E., Fowler, D. y Bebbington, P. (2002). A cognitive model of persecutory delusions. British Journal of Clinical Psychology, 41, 331-348.

Greenberg, M. S., Vazquez, C. V. y Alloy, L. B. (1988). Depression versus anxiety: Differences in self and other schemata. In L. B. Alloy (Ed.), Cognitive processes in depression (pp. 109-142). New York: Guilford Press.

Jiménez, F., Vázquez, C., y Hernangómez, L. (1998). Adjetivos en castellano de contenido depresivo autorreferente y de contenido neutral: normas de emocionalidad y frecuencia subjetiva de uso. Revista de Psicopatología y Psicología Clínica, 3, 199-215.

Juilland, A., y Chang Rodríguez, E. (1964). Frequency Dictionary of Spanish Words. La Haya: Mouton \& Co.

Kinderman, P., Prince, S., Waller, G. y Peters, E. (2003). Self-discrepances, attentional bias and persecutory delusions. British Journal of Clinical Psychology, 42,1-12.

Lang, P. J., Bradley, M. M., y Cuthbert, B. N. (1999). International affective picture system (IAPS): Technical manual and affective ratings. Gainesville, FL: The Center for Research in Psychophysiology, University of Florida.

Mathews, A. y MacLeod, C. (2005). Cognitive vulnerability to emotional disorders. Annual Review of Clinical Psychology, 1, 167-195.

McNally, R.J.; Amir, N.; Louro, C.E.; Lukach, B.M.; Riemann, B.C. y Calamari, J.E. (1994). Cognitive processing of idiographic information in panic disorder. Behaviour Research and Therapy, 32, 119-122.

Moltó, J., Montañés, S., Poy, R., Segarra, P., Pastor, M. C., Tormo, M. P., Ramírez, I., Hernández, M. A., Sánchez, M., Fernández, M. C. y Vila, J. (1999). Un nuevo método para el estudio experimental de las emociones: el International Affective Picture System (IAPS). Adaptación española. Revista de Psicología General y Aplicada, 52, 55-87.

Nieto, M., Hervás, G. y Vázquez, C. (2006). Cuestionario de Palabras de Amenaza Interpersonal. Facultad de Psicología. Universidad Complutense de Madrid.

Nieto, M. y Sotoca, A. (2004). Trastorno delirante tipo persecutorio. En J. Espada, J. Olivares y F. Méndez (Ed.), Terapia Psicológica: Casos prácticos. Madrid: Pirámide.

Pérez, M.A., Alameda, J.R. y Cuetos, F. (2003). Frecuencia, longitud y vecindad ortográfica de las palabras de 3 a 16 letras del Diccionario de la Lengua Española (RAE, 1992). Revista Española de Metodología Aplicada, 8, 1-20. Disponible en internet: http://www.psico.uniovi.es/REMA/v8n2/a1/

Pérez, M. A., Campoy, G. y Navalón, C. (2001). Indice de estudios normativos en idioma español. Revista Electrónica de Metodología Aplicada, 6, 85-105. Disponible en internet: http://www.psico.uniovi.es/v6n2/
Perona-Garcelán, S., Cuevas-Yust, C., Vallina, O y Lemos, S. (2003). Terapia cognitivo-conductual de la esquizofrenia. Guía Clínica. Madrid: Minerva Ediciones.

Pons, C. y Perpiñá, C. (1996). Palabras relacionadas con la comida y el cuerpo: Un estudio sobre la selección de material estimular con significado para utilizar en tareas experimentales en el área de los trastornos alimentarios. Revista de Psicopatología y Psicología Clinica, 1, 159-167.

Proctor, R. W. y Vu, K.L. (1999). Index of norm and rating published in the Psychonomic Society journals. Behavior Research, Methods, Instruments, \& Computers, 31, 659-667.

Quero, S., Baños, R.M. y Botella, C. (2000). Sesgos cognitivos en el trastorno de pánico: comparación entre el Stroop computerizado y con tarjetas. Psicothema, 12, 165-170.

Redondo, J., Fraga, I., Comesaña, M. y Perea, M. (2005). Estudio normativo del valor afectivo de 478 palabras españolas. Psicológica, 26, 317-326. (Disponible en: http://www.uv.es/psicologica/preprints/).

Ruiz-Vargas, J.M. y Ochoa, E.F.L. (1987). Memoria. En J.M. Ruiz-Vargas (Ed,), Esquizofrenia: Un enfoque cognitivo (pp. 165-208).. Madrid: Alianza

Ruipérez, M. A. y Belloch, A. (1997). Depresión y autoesquemas depresivos en pacientes deprimidos y ansiosos. Revista de Psicopatología Clínica y Psicología Clínica, 2, 65-80.

Sanz, J. (1995). Estímulos verbales relacionados con la ansiedad social y la depresión para uso en investigaciones cognitivas. Análisis y Modificación de Conducta, 21, 483-515.

Sanfeliu, M. C. y Fernández, A. (1996). A set of 254 Snodgrass-Vanderwart pictures standardized for Spanish: Norms for name agreement, image agreement, familiarity, and visual complexity. Behavior Research Methods, Instruments and Computers, 28 , 537-555.

Sebastián, N., Martí, M. A., Carreiras, M. y Cuetos, F. (2000). LEXESP. Léxico informatizado del español. Barcelona: Edicions Universitat de Barcelona.

Vázquez, C. y Cameron, C. (1997). Taxonomía cognitiva, psicopatología, y psicoterapias cognitivas. En I. Caro (ed.), Manual de Psicoterapia Cognitiva (pp. 53-70). Barcelona: Paidos.

Vázquez, C., Fuentenebro, F., Sanz, J., Gómez, I., Calcedo, A., Ochoa, E.F.L., \& Cerviño, M.J. (1990). Attentional performance and positive vs. negative symptoms in schizophrenia. En P.J. Drenth, J.A. Sergeant, \& J. Takens (Eds.), European Perspectives of Psychology (Vol. 3, pp. 91-106). New York: John Wiley. 
Palabras de contenido paranoide en castellano

Anexo I. Listado de palabras y estadísticos descriptivos respecto a su grado de contenido amenazante y contenido depresivo

\begin{tabular}{|c|c|c|c|c|c|c|c|c|c|}
\hline \multicolumn{10}{|c|}{ Contenido } \\
\hline \multirow[b]{2}{*}{ Palabra } & \multicolumn{2}{|c|}{ Amenaza } & \multicolumn{2}{|c|}{ Depresivo } & \multirow[b]{2}{*}{ Palabra } & \multicolumn{2}{|c|}{ Amenaza } & \multicolumn{2}{|c|}{ Depresivo } \\
\hline & Media & $D T$ & Media & $D T$ & & Media & $D T$ & Media & $D T$ \\
\hline Abandonado/a & 5,51 & 2,88 & 7,77 & 1,67 & Desconectado/a & 3,00 & 2,58 & 4,71 & 2,86 \\
\hline Abatido/a & 3,70 & 2,61 & 7,50 & 1,84 & Desconsolado/a & 3,75 & 2,94 & 7,51 & 1,79 \\
\hline Abucheado/a & 6,90 & 2,45 & 5,84 & 2,64 & Desesperanzado/a & 4,42 & 3,09 & 8,22 & 1,65 \\
\hline Aburrido/a & 2,30 & 2,43 & 4,72 & 2,80 & Desganado/a & 2,66 & 2,40 & 7,30 & 2,07 \\
\hline Acabado/a & 6,22 & 2,78 & 7,53 & 2,17 & Desgraciado/a & 5,35 & 2,99 & 8,03 & 1,67 \\
\hline Acosado/a & 7,78 & 2,36 & 3,91 & 3,01 & Desilusionado/a & 3,90 & 2,84 & 7,79 & 1,82 \\
\hline Acusado/a & 7,09 & 2,30 & 4,23 & 2,86 & Desmotivado/a & 3,83 & 2,78 & 7,67 & 2,16 \\
\hline Aislado/a & 3,51 & 2,58 & 7,41 & 1,90 & Desnutrido/a & 5,48 & 3,48 & 4,46 & 3,03 \\
\hline Aislado/a & 5,69 & 2,93 & 7,48 & 1,99 & Desolado/a & 4,57 & 2,99 & 7,72 & 1,87 \\
\hline Alarmado/a & 4,69 & 2,72 & 3,28 & 2,57 & Desorientado/a & 3,95 & 2,79 & 4,81 & 2,76 \\
\hline Alicaído/a & 3,09 & 2,49 & 7,27 & 2,13 & Desplome & 4,28 & 3,01 & 6,35 & 2,61 \\
\hline Amenazado/a & 7,81 & 2,40 & 4,09 & 2,83 & Despreciable & 5,95 & 3,20 & 4,68 & 3,06 \\
\hline Angustia & 6,14 & 2,86 & 7,18 & 2,46 & Despreciado/a & 6,80 & 2,85 & 6,36 & 2,66 \\
\hline Angustiado/a & 6,35 & 2,69 & 7,36 & 2,35 & Desprestigiado/a & 6,45 & 2,46 & 5,51 & 2,81 \\
\hline Apagado/a & 2,91 & 2,43 & 7,20 & 2,24 & Desprotegido/a & 6,26 & 2,50 & 5,93 & 2,66 \\
\hline Apenado/a & 3,30 & 2,70 & 7,81 & 1,83 & Destruir & 6,30 & 2,92 & 3,45 & 3,13 \\
\hline Apesadumbrado/a & 2,93 & 2,50 & 7,44 & 1,92 & Destruido/a & 7,01 & 2,77 & 5,95 & 3,03 \\
\hline Apresado/a & 6,72 & 2,77 & 4,49 & 2,90 & Desvalido/a & 5,33 & 2,71 & 6,71 & 2,31 \\
\hline Asediado/a & 6,73 & 2,64 & 4,38 & 2,72 & Difamado/a & 6,35 & 2,23 & 4,93 & 2,67 \\
\hline Asesinar & 9,03 & 1,93 & 3,65 & 3,39 & Disimulado/a & 2,48 & 2,45 & 2,55 & 2,52 \\
\hline Asesinado/a & 9,16 & 1,96 & 3,88 & 3,62 & Disminuido/a & 4,08 & 3,05 & 4,84 & 2,91 \\
\hline Atacado/a & 8,35 & 2,16 & 4,25 & 3,06 & Dolor & 6,88 & 2,87 & 6,36 & 2,76 \\
\hline Atemorizado/a & 7,66 & 2,01 & 5,26 & 2,97 & Enfermo/a & 6,07 & 3,01 & 6,14 & 2,90 \\
\hline Atracado/a & 8,03 & 2,06 & 3,60 & 3,14 & Engañado/a & 6,48 & 2,53 & 4,57 & 3,00 \\
\hline Bloqueado/a & 4,66 & 2,47 & 5,00 & 2,67 & Envidiado/a & 3,93 & 2,69 & 1,92 & 2,29 \\
\hline Boicoteado/a & 6,42 & 2,23 & 4,16 & 2,57 & Espiado/a & 6,20 & 2,73 & 1,98 & 2,44 \\
\hline Burlado/a & 6,27 & 2,30 & 4,75 & 2,79 & Estafado/a & 6,06 & 2,63 & 2,71 & 2,67 \\
\hline Calumniado/a & 6,68 & 2,54 & 4,68 & 2,81 & Estorbo & 4,95 & 3,19 & 5,91 & 3,02 \\
\hline Capturado/a & 7,22 & 2,58 & 4,14 & 3,00 & Estúpido/a & 5,41 & 3,26 & 4,72 & 3,38 \\
\hline Castigado/a & 6,30 & 2,57 & 4,23 & 2,79 & $\mathrm{Feo} / \mathrm{a}$ & 4,50 & 3,39 & 4,85 & 3,37 \\
\hline Chantajeado/a & 6,42 & 2,51 & 3,33 & 2,69 & Fingido/a & 4,00 & 3,11 & 2,48 & 2,70 \\
\hline Condenado/a & 7,80 & 2,38 & 4,95 & 3,05 & Flojo/a & 3,23 & 2,62 & 4,90 & 2,77 \\
\hline Controlado/a & 6,42 & 2,27 & 3,80 & 2,83 & Fracasado/a & 6,28 & 2,81 & 7,59 & 2,07 \\
\hline Criticado/a & 5,93 & 2,66 & 5,05 & 2,84 & Fracaso & 5,98 & 2,79 & 6,91 & 2,46 \\
\hline Culpable & 6,59 & 2,69 & 6,08 & 2,96 & Herido/a & 6,59 & 2,28 & 5,68 & 2,89 \\
\hline Culpado/a & 7,06 & 2,44 & 6,00 & 2,88 & Humillado/a & 7,41 & 2,31 & 6,41 & 2,73 \\
\hline Dañado/a & 7,08 & 2,31 & 6,20 & 2,81 & Hundido/a & 6,27 & 2,92 & 8,34 & 1,68 \\
\hline Débil & 4,43 & 2,83 & 6,41 & 2,58 & Ido/a & 3,50 & 2,97 & 4,28 & 2,83 \\
\hline Decaído/a & 2,95 & 2,36 & 7,83 & 2,03 & Impedido/a & 5,27 & 2,93 & 5,23 & 2,79 \\
\hline Dependiente & 4,16 & 2,78 & 5,83 & 2,48 & Impotente & 5,78 & 2,88 & 5,71 & 2,88 \\
\hline Deprimido/a & 4,56 & 3,24 & 9,54 & 0,91 & Inactivo/a & 3,75 & 2,94 & 6,06 & 2,90 \\
\hline Derrotado/a & 5,32 & 3,07 & 8,06 & 1,64 & Inadecuado/a & 3,84 & 2,88 & 3,90 & 2,93 \\
\hline Derrumbado/a & 5,16 & 3,14 & 7,70 & 2,17 & Inapetente & 2,97 & 2,51 & 5,98 & 2,73 \\
\hline Desafiado/a & 5,13 & 2,70 & 2,79 & 2,50 & Incapaz & 5,30 & 2,90 & 6,17 & 2,49 \\
\hline Desalentado/a & 3,90 & 2,47 & 6,51 & 2,17 & Incompetente & 5,88 & 2,89 & 5,40 & 3,02 \\
\hline Desamparado/a & 4,89 & 2,90 & 7,36 & 1,84 & Indeciso/a & 3,51 & 2,84 & 4,11 & 2,99 \\
\hline
\end{tabular}


Anexo I (continuación)

\begin{tabular}{|c|c|c|c|c|c|c|c|c|c|}
\hline \multicolumn{10}{|c|}{ Contenido } \\
\hline \multirow[b]{2}{*}{ Palabra } & \multicolumn{2}{|c|}{ Amenaza } & \multicolumn{2}{|c|}{ Depresivo } & \multirow[b]{2}{*}{ Palabra } & \multicolumn{2}{|c|}{ Amenaza } & \multicolumn{2}{|c|}{ Depresivo } \\
\hline & Media & $D T$ & Media & $D T$ & & Media & $D T$ & Media & $D T$ \\
\hline Indiferente & 2,94 & 2,88 & 3,86 & 3,24 & Plagiado/a & 4,55 & 3,01 & 2,55 & 2,60 \\
\hline Indigno/a & 5,02 & 2,92 & 4,08 & 3,24 & Pobre & 4,72 & 3,02 & 3,93 & 3,07 \\
\hline Infeliz & 6,02 & 3,24 & 8,53 & 1,68 & Rechazado/a & 6,70 & 2,29 & 6,76 & 2,32 \\
\hline Inferior & 5,90 & 3,14 & 6,87 & 2,71 & Renegado/a & 5,19 & 3,04 & 5,23 & 3,20 \\
\hline Inhibido/a & 4,16 & 2,97 & 4,82 & 2,97 & Reprimido/a & 4,84 & 2,81 & 4,85 & 2,80 \\
\hline Injuriado/a & 5,74 & 2,83 & 4,13 & 3,03 & Restringido/a & 4,76 & 2,83 & 3,77 & 2,97 \\
\hline Insensible & 4,32 & 3,16 & 2,85 & 2,89 & Retado/a & 5,08 & 2,88 & 2,19 & 2,41 \\
\hline Inservible & 5,70 & 2,98 & 6,24 & 3,02 & Ridiculizado/a & 7,05 & 2,35 & 5,43 & 3,20 \\
\hline Insignificante & 5,41 & 3,05 & 6,23 & 2,88 & Sangre & 5,51 & 3,59 & 1,66 & 2,41 \\
\hline Insultado/a & 6,49 & 2,75 & 5,20 & 3,07 & Secuestrado/a & 8,06 & 2,55 & 4,23 & 3,48 \\
\hline Inútil & 6,20 & 2,86 & 6,55 & $\begin{array}{l}2,85 \\
295\end{array}$ & Simulado/a & 3,40 & 2,63 & 2,10 & 2,33 \\
\hline $\begin{array}{l}\text { Lisiado/a } \\
\text { Malo/a }\end{array}$ & $\begin{array}{l}5,73 \\
6,24\end{array}$ & $\begin{array}{l}3,06 \\
2,85\end{array}$ & $\begin{array}{l}4,41 \\
2,85\end{array}$ & $\begin{array}{l}2,95 \\
277\end{array}$ & Solitario/a & 3,86 & 2,97 & 6,97 & 2,58 \\
\hline $\begin{array}{l}\text { Malo/a } \\
\text { Maltratado/a }\end{array}$ & $\begin{array}{l}6,24 \\
8,08\end{array}$ & $\begin{array}{l}2,85 \\
2,53\end{array}$ & $\begin{array}{l}2,85 \\
6,36\end{array}$ & $\begin{array}{l}2,77 \\
3,30\end{array}$ & Solo/a & 5,00 & 3,04 & 7,98 & 1,84 \\
\hline Manipulado/a & 6,93 & 2,62 & 4,52 & $\begin{array}{l}3,50 \\
2,92\end{array}$ & Sombrio/a & 4,39 & 2,81 & 6,01 & 2,94 \\
\hline Marginado/a & 7,00 & 2,70 & 6,40 & 2,97 & Subordinado/a & 4,01 & 2,73 & 3,72 & 3,01 \\
\hline Matar & 9,13 & 1,92 & 3,79 & 3,74 & Sufrimiento & 7,59 & 2,18 & 8,36 & 1,84 \\
\hline Mediocre & 5,03 & 2,90 & 4,90 & 3,12 & Suicida & 8,15 & 2,68 & 7,92 & 2,76 \\
\hline Mísero/a & 5,17 & 2,80 & 5,43 & 3,11 & Traicionado/a & 7,47 & 2,14 & 5,88 & 2,93 \\
\hline Perdedor /a & 6,17 & 2,94 & 7,00 & 2,37 & Triste & 4,83 & 3,13 & 8,65 & 1,67 \\
\hline Perdido/a & 4,93 & 2,83 & 5,81 & 2,87 & Vacío/a & 5,49 & 3,24 & 8,26 & 2,16 \\
\hline Perjudicado/a & 5,63 & 2,39 & 5,03 & 2,78 & Vapuleado/a & 6,44 & 2,28 & 5,28 & 2,81 \\
\hline Perseguido/a & 6,90 & 2,58 & 3,55 & 2,84 & Vencido/a & 6,11 & 2,63 & 6,83 & 2,45 \\
\hline Pesimista & 4,20 & 2,94 & 7,70 & 2,50 & Vigilado/a & 6,30 & 2,79 & 2,75 & 2,62 \\
\hline
\end{tabular}


Anexo II. Listado de palabras y estadísticos descriptivos (puntuaciones medias) en las variables negatividad (emocionalidad negativa) y frecuencia de uso.

\begin{tabular}{|c|c|c|c|c|c|c|c|c|c|}
\hline & \multicolumn{2}{|c|}{ Negatividad } & \multicolumn{2}{|c|}{ Frecuencia } & & \multicolumn{2}{|c|}{ Negatividad } & \multicolumn{2}{|c|}{ Frecuencia } \\
\hline & Media & $D T$ & Media & $D T$ & & Media & $D T$ & Media & $D T$ \\
\hline Abandonado/a & 7,40 & 2,02 & 3,52 & 2,30 & Desconsolado/a & 6,16 & 1,83 & 3,29 & 2,27 \\
\hline Abatido/a & 6,14 & 2,22 & 2,31 & 2,36 & Desesperanzado/a & 7,33 & 1,89 & 3,64 & 2,49 \\
\hline Abucheado/a & 6,86 & 2,16 & 1,77 & 1,77 & Desganado/a & 5,41 & 2,15 & 5,64 & 2,31 \\
\hline Aburrido/a & 5,16 & 2,10 & 6,83 & 2,17 & Desgraciado/a & 7,42 & 1,95 & 4,32 & 2,99 \\
\hline Acabado/a & 7,78 & 1,93 & 3,34 & 2,70 & Desilusionado/a & 6,74 & 2,11 & 5,67 & 2,57 \\
\hline Acosado/a & 7,44 & 2,04 & 2,23 & 2,20 & Desmotivado/a & 6,25 & 1,99 & 5,73 & 2,61 \\
\hline Acusado/a & 7,40 & 9,33 & 2,51 & 2,27 & Desnutrido/a & 7,14 & 2,48 & 2,18 & 2,04 \\
\hline Afligido/a & 5,95 & 1,89 & 1,87 & 1,96 & Desolado/a & 7,50 & 9,11 & 2,48 & 2,16 \\
\hline Aislado/a & 6,85 & 2,09 & 3,33 & 2,32 & Desorientado/a & 5,05 & 1,87 & 4,11 & 2,25 \\
\hline Alarmado/a & 4,75 & 2,06 & 2,75 & 2,36 & Desplome & 5,68 & 2,37 & 1,51 & 1,64 \\
\hline Alicaído/a & 4,98 & 1,81 & 2,22 & 2,93 & Despreciable & 7,53 & 2,12 & 3,40 & 2,48 \\
\hline Amenazado/a & 7,28 & 2,09 & 3,12 & 2,47 & Despreciado/a & 7,72 & 1,92 & 3,57 & 3,73 \\
\hline Angustia & 7,61 & 2,02 & 5,15 & 2,77 & Desprestigiado/a & 6,24 & 1,87 & 2,42 & 2,14 \\
\hline Angustiado/a & 7,64 & 1,87 & 5,18 & 2,92 & Desprotegido/a & 6,57 & 1,95 & 3,78 & 5,89 \\
\hline Apagado/a & 5,30 & 1,88 & 5,43 & 2,32 & Destruir & 6,86 & 2,32 & 4,08 & 2,76 \\
\hline Apenado/a & 6,15 & 1,80 & 4,06 & 2,35 & Destruido/a & 7,43 & 2,03 & 3,77 & 2,63 \\
\hline Apesadumbrado/a & 5,50 & 1,76 & 1,33 & 1,80 & Desvalido/a & 6,61 & 1,84 & 2,27 & 1,95 \\
\hline Apresado/a & 6,20 & 2,30 & 1,12 & 1,50 & Difamado/a & 6,05 & 2,02 & 1,89 & 1,77 \\
\hline Asediado/a & 6,43 & 2,00 & 1,15 & 1,51 & Disimulado/a & 2,60 & 2,04 & 3,77 & 2,56 \\
\hline Asesinar & 9,10 & 1,91 & 3,37 & 2,95 & Disminuido/a & 4,83 & 2,80 & 2,92 & 2,43 \\
\hline Asesinado/a & 9,34 & 1,69 & 3,52 & 2,99 & Dolor & 7,83 & 2,14 & 6,99 & 2,32 \\
\hline Atacado/a & 7,89 & 1,76 & 4,49 & 2,64 & Enfermo/a & 7,33 & 2,24 & 6,60 & 2,49 \\
\hline Atemorizado/a & 7,69 & 1,78 & 3,87 & 2,54 & Engañado/a & 7,30 & 1,92 & 5,91 & 2,58 \\
\hline Atracado/a & 7,75 & 1,88 & 3,19 & 2,68 & Envidiado/a & 5,39 & 2,67 & 5,18 & 2,40 \\
\hline Bloqueado/a & 5,80 & 2,13 & 5,31 & 2,36 & Espiado/a & 6,32 & 5,58 & 2,92 & 2,27 \\
\hline Boicoteado/a & 6,13 & 1,93 & 1,87 & 1,74 & Estafado/a & 6,33 & 2,04 & 3,76 & 9,55 \\
\hline Burlado/a & 6,32 & 2,06 & 2,87 & 2,40 & Estorbo/a & 6,61 & 2,17 & 3,61 & 2,51 \\
\hline Calumniado/a & 6,68 & 2,14 & 2,16 & 2,10 & Estúpido/a & 6,77 & 2,02 & 5,52 & 3,04 \\
\hline Capturado/a & 6,86 & 2,36 & 1,79 & 1,72 & Feo/a & 5,71 & 2,22 & 6,38 & 2,77 \\
\hline Castigado/a & 6,05 & 2,00 & 5,20 & 2,46 & Flojo/a & 4,51 & 2,17 & 4,31 & 2,77 \\
\hline Chantajeado/a & 6,48 & 2,04 & 3,49 & 2,45 & Fingido/a & 4,54 & 2,25 & 2,90 & 2,42 \\
\hline Condenado/a & 7,42 & 1,97 & 3,15 & 2,51 & Fracasado/a & 7,39 & 1,85 & 4,35 & 2,71 \\
\hline Controlado/a & 6,18 & 2,22 & 6,40 & 10,65 & Fracaso/a & 7,53 & 6,15 & 4,52 & 2,68 \\
\hline Criticado/a & 6,25 & 1,98 & 6,15 & 2,41 & Herido/a & 7,46 & 9,12 & 4,81 & 2,68 \\
\hline Culpable & 7,13 & 2,18 & 5,90 & 2,46 & Humillado/a & 7,52 & 1,74 & 4,37 & 2,82 \\
\hline Culpado/a & 7,14 & 2,01 & 4,35 & 2,64 & Hundido/a & 7,43 & 1,82 & 4,35 & 2,73 \\
\hline Dañado/a & 7,10 & 2,00 & 3,99 & 2,38 & Ido/a & 4,69 & 2,14 & 4,79 & 2,91 \\
\hline Débil & 6,45 & 1,96 & 5,95 & 2,68 & Impedido/a & 5,86 & 2,33 & 1,97 & 2,05 \\
\hline Decaído/a & 5,99 & 1,86 & 5,40 & 2,55 & Impotente & 6,46 & 1,99 & 4,68 & 6,61 \\
\hline Dependiente & 5,96 & 2,41 & 5,88 & 2,58 & Inactivo/a & 5,13 & 2,22 & 3,85 & 2,54 \\
\hline Deprimido/a & 7,93 & 3,66 & 6,31 & 2,55 & Inadecuado/a & 4,41 & 2,13 & 3,74 & 2,63 \\
\hline Derrotado/a & 7,33 & 1,99 & 4,57 & 10,93 & Inapetente & 4,16 & 1,96 & 2,01 & 2,09 \\
\hline Derrumbado/a & 6,93 & 2,21 & 2,93 & 2,53 & Incapaz & 6,00 & 2,07 & 4,89 & 2,69 \\
\hline Desafiado/a & 4,63 & 1,81 & 2,59 & 2,25 & Incompetente & 6,34 & 1,96 & 4,16 & 2,82 \\
\hline Desalentado/a & 5,60 & 1,73 & 2,12 & 1,91 & Indeciso/a & 4,90 & 2,21 & 6,04 & 2,39 \\
\hline Desamparado/a & 6,69 & 1,90 & 2,30 & 2,18 & Indiferente & 5,27 & 6,72 & 5,23 & 2,52 \\
\hline Desconectado/a & 3,88 & 1,95 & 3,95 & 2,54 & Indigno/a & 5,84 & 2,21 & 2,21 & 2,04 \\
\hline
\end{tabular}


Anexo II (continuación)

\begin{tabular}{|c|c|c|c|c|c|c|c|c|c|}
\hline & \multicolumn{2}{|c|}{ Negatividad } & \multicolumn{2}{|c|}{ Frecuencia } & & \multicolumn{2}{|c|}{ Negatividad } & \multicolumn{2}{|c|}{ Frecuencia } \\
\hline & Media & $D T$ & Media & $D T$ & & Media & $D T$ & Media & $D T$ \\
\hline Infeliz & 8,26 & 1,86 & 4,82 & 3,06 & Perjudicado/a & 5,63 & 1,93 & 4,43 & 2,71 \\
\hline Inferior & 14,51 & 79,98 & 4,61 & 3,03 & Perseguido/a & 6,25 & 2,18 & 3,08 & 2,40 \\
\hline Inhibido/a & 5,18 & 2,06 & 3,22 & 2,48 & Pesimista & 6,46 & 2,31 & 6,31 & 2,36 \\
\hline Injuriado/a & 5,92 & 1,79 & 1,54 & 1,91 & Plagiado/a & 5,24 & 2,20 & 2,33 & 2,17 \\
\hline Insensible & 6,75 & 2,06 & 5,10 & 7,58 & Pobre & 5,64 & 2,52 & 5,58 & 2,79 \\
\hline Inservible & 6,57 & 2,14 & 3,50 & 2,59 & Rechazado/a & 6,96 & 1,82 & 4,82 & 2,77 \\
\hline Insignificante & 6,45 & 2,07 & 3,79 & 2,62 & Renegado/a & 5,50 & 2,38 & 2,35 & 2,20 \\
\hline Insultado/a & $\begin{array}{r}6,59 \\
735\end{array}$ & $\begin{array}{l}1,99 \\
1,02\end{array}$ & $\begin{array}{l}4,51 \\
4,95\end{array}$ & $\begin{array}{l}2,88 \\
2,90\end{array}$ & Reprimido/a & 5,72 & 2,21 & 4,17 & 2,63 \\
\hline $\begin{array}{l}\text { Inútil } \\
\text { Lisiado/a }\end{array}$ & $\begin{array}{l}7,35 \\
6,39\end{array}$ & $\begin{array}{l}1,93 \\
4,28\end{array}$ & $\begin{array}{l}4,95 \\
1,78\end{array}$ & $\begin{array}{l}2,90 \\
2,13\end{array}$ & Restringido/a & 5,10 & 2,22 & 2,68 & 2,25 \\
\hline Malo/a & 7,18 & 2,43 & 6,43 & 2,89 & Retado/a & 3,71 & 2,12 & 2,21 & 2,25 \\
\hline Maltratado/a & 8,55 & 1,95 & 4,58 & 3,06 & Ridiculizado/a & 6,61 & 2,01 & 3,56 & 2,48 \\
\hline Manipulado/a & 7,27 & 2,04 & 4,29 & 2,62 & Sangre & 5,63 & 8,73 & 5,02 & 2,94 \\
\hline Marginado/a & 7,44 & 2,07 & 4,64 & 2,64 & Secuestrado/a & 8,22 & 2,08 & 2,80 & 2,55 \\
\hline Matar & 9,18 & 1,84 & 4,97 & 3,38 & Simulado/a & 3,64 & 2,31 & 2,01 & 2,10 \\
\hline Mediocre & 6,02 & 2,17 & 3,29 & 2,63 & Solitario/a & 5,64 & 6,85 & 4,83 & 2,70 \\
\hline Mísero/a & 6,16 & 1,99 & 2,00 & 2,03 & Solo/a & 6,20 & 2,79 & 6,00 & 2,74 \\
\hline Perdedor/a & 6,42 & 2,09 & 3,66 & 2,57 & Sombrío/a & 4,96 & 2,18 & 2,99 & 5,54 \\
\hline Perdido/a & 5,45 & 2,26 & 4,56 & 2,68 & Subordinado/a & 4,82 & 1,98 & 2,29 & 2,27 \\
\hline
\end{tabular}

